\title{
Somatic manifestations in renal disease: A clinical research study
}

\author{
WILLIAM L. JOHNSTON, D.O., FAAO \\ East Lansing, Michigan \\ ALBERT F. KELSO, PH.D. \\ DONALD L. HOLLANDSWORTH, D.O \\ JOHN J. KARRAT, D.O. \\ Chicago, Illinois
}

This study reports on clinically useful signs in patients with renal disease. In a controlled clinical trial, three groups of patients were examined to test the assumption that somatic manifestations of renal disease would be present in the spinal region T9-12. One group of patients had advanced renal disease; the two control groups consisted of hypertensive and normotensive patients without signs of renal disease. Patients were excluded from the study if they had other conditions that would cause similar findings. Recorded findings of both palpatory examination and thermography of the thoracic spinal region revealed a significantly higher frequency of segmental dysfunction and areas of elevated skin temperature in the region T9-12 for the renal group. These data support the presence of somatic motor and vasomotor changes as reflex components of renal disease. The clinician who uses reflex findings in this spinal region to assist in identification of renal disease will need to give diagnostic consideration to other sources of findings, including such falsepositives as somatic and nonrenal visceral stimuli.

Renal disease creates renal reflexes when the disease process involves inflammation, chemical changes in the kidney's internal environment, and alterations in renal sensory nerve activity. Not only do the resulting somatic components of these reflexes produce signs that are useful in physical diagnosis, but, also, lower back pain is an extremely common clinical complaint of patients with kidney disease. Although renal reflexes have been investigated extensively in animal models, there have been few studies directed toward evaluating the clinical signs of this reflex component in patients with renal disease.

There is an anatomic ${ }^{1}$ and a physiologic basis ${ }^{2,3}$ for the general assumption that visceral disease is accompanied by reflexes that cause somatic tissue changes. Inflammation, organ distention, large compression forces, spasms (if the organ is muscular), ischemia, and endogenous or exogenous chemical irritants are major sources of stimuli in diseased visceral organs. Specifically, visceral afferents from the kidney follow sympathetic nerves, passing through the ganglia of the sympathetic chain to enter the spinal cord via the dorsal root in an ipsilateral fashion; that is, right kidney afferents via right dorsal root. ${ }^{4}$ The visceral afferent cell body is located in the dorsal root ganglion, and reflex connections are established as follows: (1) at the same segmental level with somatic motor and preganglionic sympathetic neurons; (2) at adjacent segmental levels; and (3) with neurons ascending in the spinothalamic tract. The kidney receives preganglionic sympathetic fibers from the tenth thoracic through the first lumbar sympathetic ganglia, and afferent fibers from the kidney enter mainly at cord levels T10-12. ${ }^{4}$ There should be an allowance of one or two segments' deviation, because the visceral nervous system shows some variability in location and has some variation in its structural development. Reflex responses in seg- 


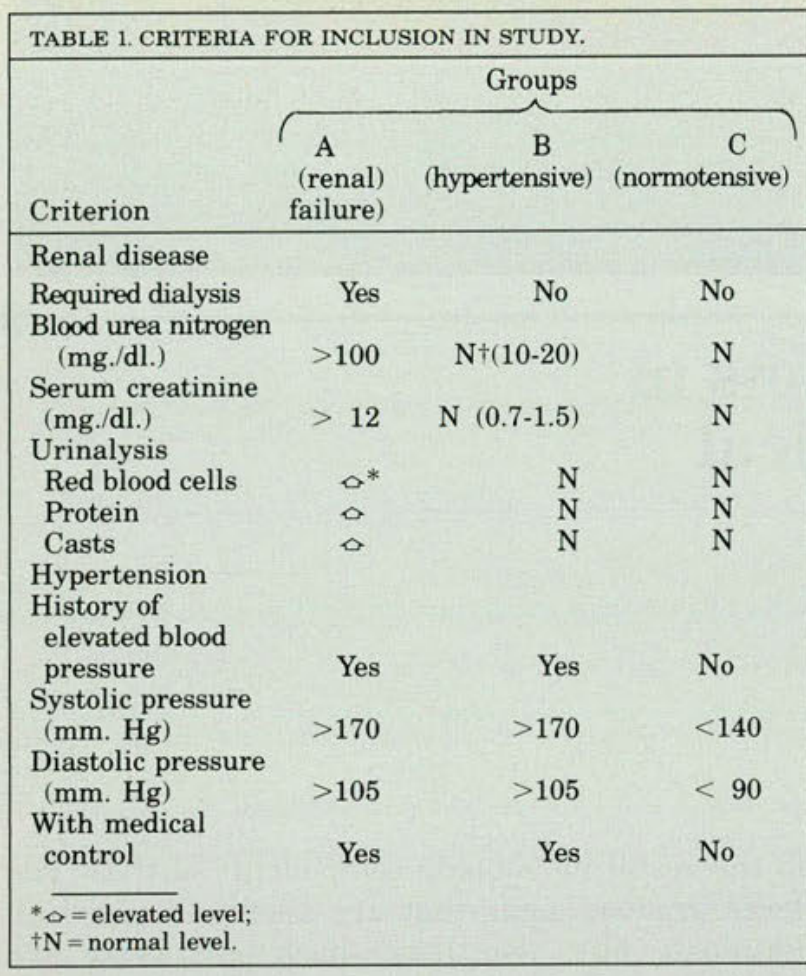

mentally related somatic tissues have been observed experimentally, both in laboratory preparations of animal models ${ }^{5}$ and with human subjects in a surgical setting. ${ }^{6-8}$ These and other clinical observations $^{2,9}$ have linked somatic manifestations of sensory, motor, and vasomotor disturbances in the somatic tissues to a number of visceral diseases.

The present study focuses on two reflex components of renal disease: (1) somatic motor changes observed.as palpable increased muscular tension and reduced mobility, as previously reported ${ }^{10}$; and (2) vasomotor changes recorded as skin temperature variations. These components were established in previous reports, ${ }^{10-12}$ which described our methods of examining the paravertebral tissues of the back and recording thermographic data.

Procedures for palpatory diagnosis of dysfunction in somatic tissues have been studied by multiple examiners..$^{10,13}$ In these studies, the examination procedures identified dysfunctions as motion limitations in spinal and costal articulations that exhibited an asymmetry in response to motion tests and an accompanying palpable increase in muscular tension. Procedures for thermographic measurement were standardized and used in the examination of dorsal skin for temperature distribution (Tsk) in normal healthy adults. ${ }^{11}$ General Electric Spectrotherm $2000^{\mathrm{TM}}$ (thermoscanner) was used in a climate control chamber. A PDP-12 computer was used to control the Spectrotherm and for data storage and analysis. This method for thermography identifies altered states of cutaneous vasomotion as differences in Tsk.

In the present controlled clinical study, we have used these standardized procedures to obtain data on the presence of muscular and vasomotor manifestations of viscerosomatic reflexes in the spinal region $\mathrm{T} 9$ to $\mathrm{T} 12$ in renal dialysis patients. Because all patients who are in renal failure have a history of hypertension, we established one control group of hypertensives and a second control group of normotensives. All control patients were without renal disease.

Our data provide knowledge about the location of somatic manifestations of renal disease. Information of this kind, as obtained through controlled clinical study, will contribute to understanding spinal reflex activity and the physical signs observed during clinical examination of patients with visceral disease. Such knowledge of reflexes supports osteopathic diagnosis. We tested the assumption that in renal disease viscerosomatic reflexes would be evident as limitation of motion and as changes in vasomotor tone in the spinal region T9-12.

\section{Method}

\section{Research design}

Subject selection. Twenty subjects with diagnosed renal failure constituted the experimental group. These patients were being treated in the dialysis unit of the Chicago Osteopathic Medical Clinic. Control groups of 20 established hypertensives and 20 normotensives, all without clinical signs of renal failure, were volunteers from the Chicago Family Medicine Clinic. The project was approved by the Institutional Review Board of CCOM. All subjects were informed about the study and signed consent forms. Patients in each group were matched for age, sex, and ethnic origin to reduce the influence of these variables in the small sample.

Criteria for inclusion in the study are summarized in Table 1 . In the renal failure group, each patient required dialysis for life support. All of these patients had a history of hypertension. Medical histories of renal dialysis patients also documented the presence of blood urea nitrogen levels $>$ $100 \mathrm{mg} . / \mathrm{dl}$., serum creatinine values $>12 \mathrm{mg} . / \mathrm{dl}$., and elevated levels of red blood cells, protein, and casts in the urine. Hypertensive and normotensive control subjects received similar evaluation and had none of these signs of renal disease. Patients in the hypertensive group without renal disease were being managed medically for their high blood pressure. Prior to medication, all patients in this control group had recorded systolic pressures $>170$ 
$\mathrm{mm} . \mathrm{Hg}$ and diastolic pressures $>105 \mathrm{~mm} . \mathrm{Hg}$. Normotensive patients had no history of elevated blood pressure, nor did any of their blood pressures exceed $140 \mathrm{~mm}$. $\mathrm{Hg}$ systolic and $90 \mathrm{~mm}$. $\mathrm{Hg}$ diastolic during clinical examination.

Criteria for exclusion from all groups were established. Patients with primary idiopathic or major secondary scoliosis were excluded, which minimized the influence of gross structural problems on tests for spinal motion and paravertebral tissue changes. To reduce physician examiner bias, a history of major surgery with a scar present on the dorsal thorax was another basis for exclusion. The presence of, or a history of, recent inflammatory disease of the pelvis or colon were other criteria for exclusion, and women using intrauterine devices also were excluded. These exclusions were necessary to minimize the influence of other sources of viscerosomatic reflexes on the thermographic or clinical findings in the lower thoracic region.

Blinding procedures. Research design to introduce a physician blind (WLJ) required the following procedures: (1) clothing of renal dialysis patients to hide surgical scars; (2) scheduling testing to provide equal probability of patients from experimental or control groups arriving for testing; and (3) withholding all medical information concerning the subject until the palpatory examination had been completed.

The renal dialysis group included patients with indwelling catheters. A long-sleeved surgical paper scrub gown and pants were used, with the gown open at the back to allow palpatory examination. The gown and pants were draped to cover the lower trunk and limbs. Renal patients usually complete dialysis between 10:00 a.m. and noon, between 2:00 p.m. and 4:00 p.m., and after 6:00 p.m. Two patients were randomly scheduled for these periods. Each day's patients were randomized to provide equal probability of dialysis or nondialysis patients appearing for examination. Because there is a high incidence of hypertension in the CCOM clinic population, many patients scheduled as normotensive patients were later reclassified as having high blood pressure. This resulted in a large group of hypertensive patients. A total of 90 patients -45 hypertensives, 24 renal dialysis patients, and 21 normotensives-were enrolled into the study. Data from only 20 subjects in each group are reported. The first renal, hypertensive, or normotensive patient who met criteria for inclusion, had no basis for exclusion, and for whom there was complete data collection was used for matching in order to minimize the influence of selection in matching patients.
Randomization procedures. Patients were scheduled for palpatory/thermographic examination on a random basis to provide the physician blind. Renal failure patients were scheduled immediately following dialysis to standardize the patients' status. All patients were scheduled during daytime periods when the renal patients might be available. Patients were instructed by the person obtaining informed consent to give no medical information to their examiner (WLJ) and to make no comments about their source or length of medical care. The examiner's first encounter with the subject was in the climate control chamber for palpatory examination of the thoracic region. Examinations were conducted prior to any access by the examiner to patient records or data.

Overview of data collection procedure. Subjects were selected from the daily schedule for clinic patients and from the patients scheduled for dialysis. These patients were interviewed, and informed consent was obtained for use of medical information, for agreement to acclimation in the climate control chamber, and for commitment of at least 1 hour for these procedures, as well as the consent needed for examinations. Subjects entered the climate control chamber, gowns and scrub pants were adjusted to allow thermal equilibrium with the environment $(24 \mathrm{C} \pm 0.5 \mathrm{C}$, relative humidity $50 \pm 10$ percent, and airflow $<1.5 \mathrm{mph}$ ); 30 minutes were allowed for equilibration. During equilibration, palpatory examination of the exposed thoracic region was completed. Research records of the examiner's findings were completed and included in the subject's file. At the end of the examination, a thermogram was recorded of the patient's back. The patient's research data were then completed (WLJ), using the patient's medical record and patient interview. Missing data were noted, and any needed tests or examinations were ordered.

\section{Measurements}

Detailed descriptions of both thermographic and palpatory examinations have been reported previously. ${ }^{10-12}$

Palpatory testing. Osteopathic examination of the exposed thoracic region used three levels of examination ${ }^{14}$-an overall screen, regional scan, and segmental definition. Selected screening tests ${ }^{15}$ for signs of somatic dysfunction provided a general impression. These were followed by regional scans of tissue texture and mobility, ${ }^{10,12}$ thus providing a basis for distinguishing location of segmental dysfunctions. Three motion tests, passive sidebend- 


\section{Vertebral}

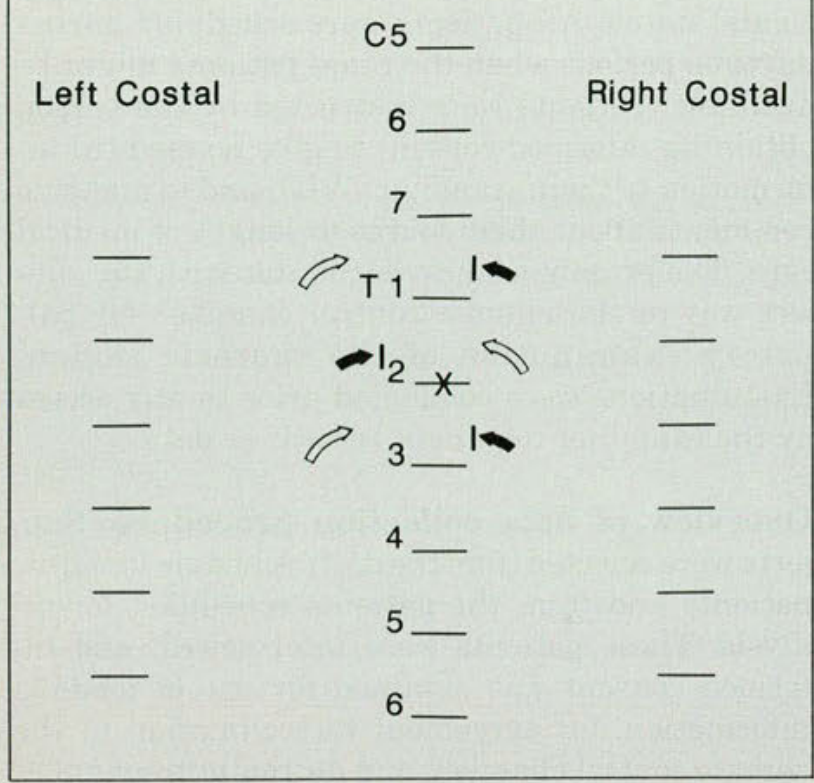

Fig. 1. Schematic representation of three mobile columns in the thoracic region, with a 3 -segment unit of dysfunction in the vertebral column. In this example, $x=$ location of the primary central segment at $T 2$, with resistance to rotation to the right (illustrated as a short, closed arrow, with a bar representing the barrier). In the adjacent segments, T1 and T3, the mirror image resistance to the left is illustrated (short, closed arrows with bars). Longer, open arrows without bars represent the sense of compliance with motion and a greater range of motion in the directions opposite to the directions of limited mobility.

ing, ${ }^{*}$ passive axial rotation, $\uparrow$ and active respiratory excursion, $\ddagger$ were then used to complete the examination; the specific features of the asymmetry in response to these 3 motion tests defined the characteristics of each dysfunctional segment.

Findings of segmental dysfunction were recorded $^{10,11}$ for each of three vertical mobile columns; palpatory contact in the midline column of vertebral segments overlies the transverse processes bilaterally, and, in the two lateral costal columns,

\footnotetext{
*For example, in testing passive sidebending to the right, the subject was seated and the examiner was standing posteriorly and to the right, facing the subject's back. While monitoring the dysfunctional segment by palpatory contact of the examiner's left hand, movement was initiated by moderate pressure of the examiner's right hand in a caudal direction on the subject's right shoulder (approximating the right shoulder toward the right hip).

†Passive axial rotation to the right was initiated by the examiner's right hand controlling introduction at the subject's right elbow (arms folded) of a rotational movement of the shoulders and trunk to the right.

$\ddagger$ The subject's active respiratory excursions, inhalation and exhalation, were directed by the examiner and monitored by palpation at the dysfunctional vertebral and costal segments. A comparison was made for the presence of more limited mobility in response to either of the opposing directions.
}

contact overlies the angle of the ribs. In previous studies, ${ }^{10,12}$ criteria were established that described a fundamental unit of segmental dysfunction as consisting of a clinically significant vertical 3-segment complex of limited movement in the vertebral and/or costal columns. The unit also exhibits a predictable pattern of signs of disturbed neuromuscular function, as follows: (1) deep, localized muscular tension, which is increased in the primary central segment in contrast to adjacent segments; (2) asymmetries in motion at this primary segment when responses to motion tests in opposing directions are compared; and (3) mirror-image asymmetries in motion in the superior and inferior adjacent segments compared to the primary segment, when responses to the same motion tests are compared. An example of the 3-segment unit of dysfunction is illustrated in the vertebral column in Figure 1; the only asymmetric motion descriptor indicated is axial rotation. We recorded the site of each primary segment (for example, at T2 in Figure 1) as the location of the segmental dysfunction.

Further, we charted primary dysfunctional segments according to their location in one of the three mobile columns (midline vertebral, right or left costal) and classified the findings at each segmental level in one of three categories. This classification of dysfunctions is based on location, on characteristics of asymmetry, and on presence or absence of linkage in dysfunction observed between adjacent mobile columns at the same segmental level.

The category of least clinical significance in our study is represented by isolated units of segmental dysfunction in either the vertebral or costal columns, that is, without the presence of any second dysfunctional unit in an adjacent column at the same segmental level. Two examples of category I are illustrated in Figure 2. One occurs in the vertebral column at T2, and another in the right costal column at rib 4.

Category II is represented by two dysfunctions that are present in adjacent columns at the same segmental level but that have dissimilar motion characteristics. For example, in Figure 2, one dysfunction occurs in the vertebral column at T6, with resistance to the axial rotation test to the right, while another dysfunction occurs in the left costal column at rib 6 , with resistance to axial rotation left.

A third category, which was both clinically and statistically significant to our study, is represented by vertebral and costal dysfunctions that are present at the same segmental level and that have identical motion asymmetries in the two adjacent columns. This category of segmental dysfunction indicates the presence of a linkage of motion 


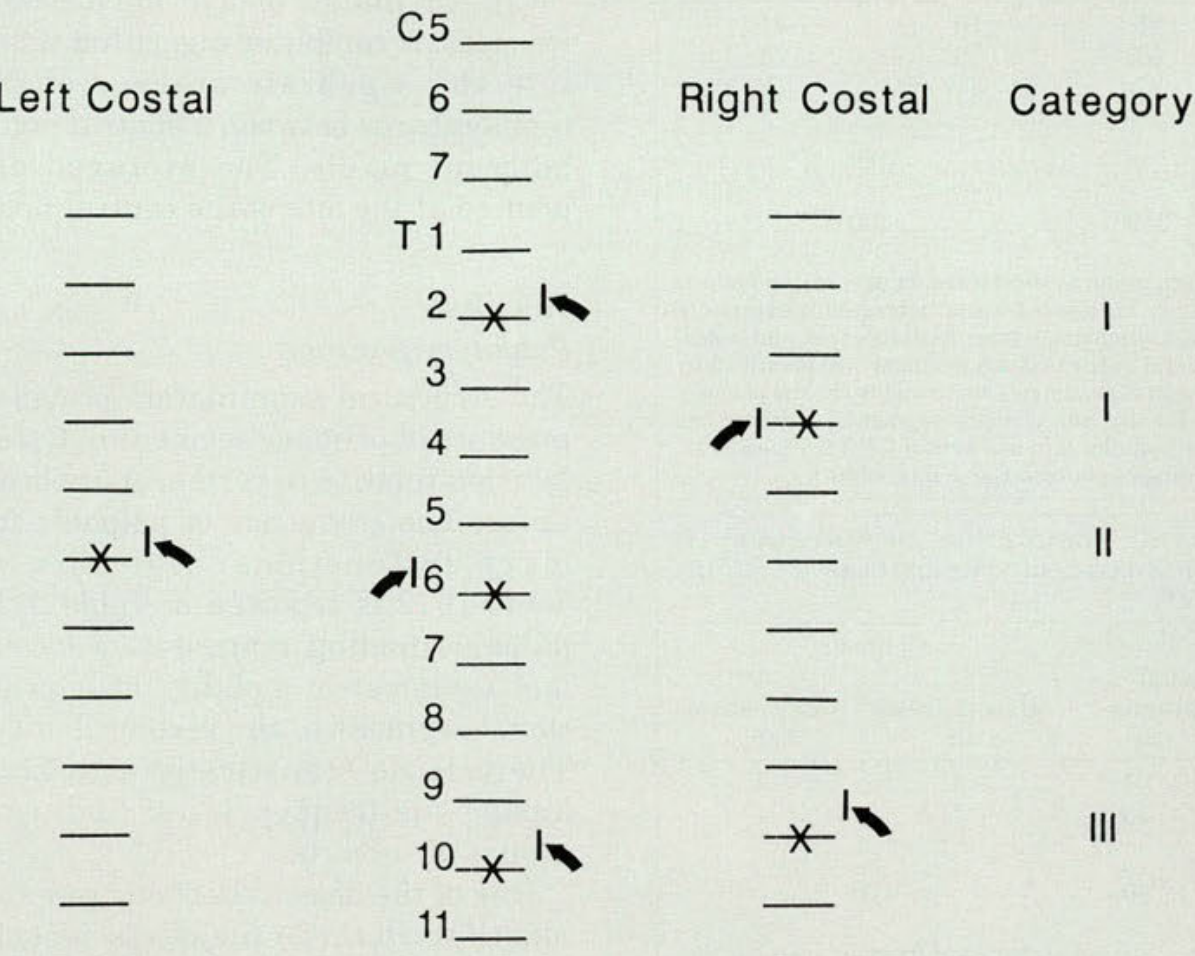

Fig. 2. Schematic representation for three categories of segmental dysfunction. Categories are based upon similarities or dissimilarities in the direction of motion restriction at primary dysfunctional segments in the vertebral column and an adjacent costal column at the same vertebral segmental level. The illustrations are for an axial rotation test. ( $X$ indicates the location of a primary dysfunctional segment.) Category I includes a single finding in any of the three mobile columns. Two illustrations are provided-resistance to axial rotation left at vertebral level T2, and resistance to axial rotation right at right rib 4. Category II includes findings at one segmental level in the midline vertebral and adjacent costal columns, with dissimilarities in direction of resistance to motion. Illustrated at vertebral level T6 are resistance to axial rotation to the right in the vertebral column and resistance to axial rotation left in the left costal column. Category III includes findings at one segmental level in the vertebral and adjacent costal columns, with similarities in the direction of resistance to motion. Illustrated at vertebral level T10 are resistances to axial rotation to the left in both the vertebral and right costal columns.

characteristics in adjacent columns in response during motion testing. An example of category III is shown in Figure 2 at thoracic level 10. Both vertebral and right costal dysfunctions are identified by resistance to an axial rotation test to the left. We have hypothesized that category III, linkage of motion asymmetries in the vertebral and one adjacent costal column at the same segmental level accompanies visceral disease.

Recording procedures. To facilitate recording the anatomic location of segmental findings, four markers (each measuring 1 square centimeter) were placed on the dorsal skin surface, with their lower borders coinciding with the tip of spinous processes $\mathrm{C} 7, \mathrm{~T} 4, \mathrm{~T} 8$, and T12. A 6 " by $18^{\prime \prime}$ cloth overlay was placed over the exposed back and was used to record the locations of markers and the palpable findings of segmental dysfunction. Prior to temperature measurement, small squares of reflecting foil were placed over the markers for identifying the marker placements on the thermogram. In this manner, the two sets of data could be correlated through reference to the markers in each record.

Instrumental procedures. With the subject standing at a distance from the camera that produced a standard-sized image for all subjects, a thermoscan was recorded from the exposed dorsal skin surface. This was accomplished by matching the $\mathrm{C} 7$ and $\mathrm{T} 12$ vertebral markers to marks on the video screen for thermographic imaging. This method results in 40 rows and 20 columns of pixels 


\begin{tabular}{|c|c|c|c|}
\hline \multirow[b]{2}{*}{$\begin{array}{l}\text { Primary } \\
\text { dysfunctional } \\
\text { segments }\end{array}$} & \multirow[b]{2}{*}{$\begin{array}{l}\text { Renal } \\
\text { patients } \\
(20)\end{array}$} & \multicolumn{2}{|c|}{ Controls } \\
\hline & & $\begin{array}{l}\text { Hypertensives } \\
\text { (20) }\end{array}$ & $\begin{array}{l}\text { Normotensives } \\
(20)\end{array}$ \\
\hline Vertebral & 25 & 16 & 21 \\
\hline Costal & 37 & 35 & 23 \\
\hline Totals & $\overline{62}$ & $\overline{51}$ & $\overline{44}$ \\
\hline $\begin{array}{l}\text { Possible } \\
\text { counts }\end{array}$ & 240 & \multicolumn{2}{|c|}{480} \\
\hline \multicolumn{4}{|c|}{$\begin{array}{l}\text { *Twenty subjects in each group were matched for age, sex, and ethnic } \\
\text { group. Palpatory findings for tissue texture (increased resistance to } \\
\text { pressure) and motion asymmetries were first observed and noted } \\
\text { Then the segmental level of the primary segment was identified by } \\
\text { presence of mirror-image asymmetries above and below the primary } \\
\text { segment and noted. Finally, the primary segmental location was } \\
\text { identified by counting from the skin markers at C7, T4, T8, and T12, } \\
\text { and the palpatory findings were recorded and tabulated. }\end{array}$} \\
\hline \multicolumn{4}{|c|}{$\begin{array}{l}\text { TABLE 3. OBSERVED FREQUENCY OF CATEGORY II } \\
\text { (NONLINKAGE) AND CATEGORY III (LINKAGE) CLASSIFICATIONS } \\
\text { IN REGION T9-10-11-12.* }\end{array}$} \\
\hline \multirow[b]{2}{*}{ Classification } & \multirow[b]{2}{*}{$\begin{array}{l}\text { Renal } \\
\text { patients } \\
\qquad(20)\end{array}$} & \multicolumn{2}{|c|}{ Controls } \\
\hline & & $\begin{array}{l}\text { Hypertensives } \\
\text { (20) }\end{array}$ & $\begin{array}{l}\text { Normotensives } \\
(20)\end{array}$ \\
\hline Category II & 4 & 4 & 4 \\
\hline Category III & 13 & 4 & 1 \\
\hline Possible counts & 80 & & \\
\hline \multicolumn{4}{|c|}{$\begin{array}{l}\text { Chi square }=13.2 ; p=\langle 0.005 ; 1 \text { degree of freedom, no correction } \\
\text { factor, one-tailed test. } \\
\text { *Vertebral and costal findings occurring at the same segmental leve } \\
\text { were examined for similarity in directions of resistance to motion } \\
\text { (Category III, linkage), or dissimilarity (Category II, nonlinkage) and } \\
\text { recorded. In the one instance (in the renal group) that linkage oc } \\
\text { curred on one side but not on the other, when accompanying costa } \\
\text { findings were present bilaterally, the findings at that spinal level were } \\
\text { assessed as Category III. (This avoided counting the vertebral dys- } \\
\text { function twice.) }\end{array}$} \\
\hline
\end{tabular}

(picture elements) representing an 8 " wide exposed area from $\mathrm{C} 7$ to T12 on the patient's back. We have separately tested the effects on Tsk of the tape markers, transient skin contacts, and fingertip pressures involved in the palpatory examination. No persisting influence on skin temperature patterns could be determined even 30 seconds following the palpatory examinations.

Thermograms were recorded with a General Electric Thermoscan. Using a PDP-12 computer, the video output was controlled, converted from an analog signal to a digital signal, and the digital data were stored. The computer printouts included the following data:

(1) A symbolic image of temperature. A symbol for each $0.5 \mathrm{C}$ range was assigned to temperatures between 27 and $33 \mathrm{C}$. The symbol was then used in the printout to represent each measured skin temperature.
(2) A digital image of temperature. Eight measurements of skin temperature, four in one row and four adjacent temperatures from the row below, were averaged by a computer algorithm and printed as a pixel temperature.

(3) An image of averaged temperature differences. A computer algorithm was used to calculate the algebraic average of differences in temperatures between a central pixel and the four adjacent pixels. The averaged difference was printed at the site of the central pixel.

\section{Results \\ Palpatory findings}

The structural examination provided data on the presence of primary segmental dysfunction and its location relative to vertebral levels and mobile columns. The frequency of palpable findings of primary dysfunctional segments in the region T9-10-11-12 is reported in Table 2. Each recorded palpable finding represents a location of limited and asymmetric mobility at a primary dysfunctional segment in the vertebral or costal columns. There is no statistically significant difference among the frequencies of findings in the three groups of subjects.

One of the objectives of our research has been to identify criteria for the assessment of differences in findings related to the etiology of somatic dysfunction; that is, is the dysfunction related to stimuli of somatic or visceral origin? In this study, renal disease has been addressed as a primary question, and the palpatory findings were evaluated as related response variables. It is possible to have limited mobility occurring in midline or lateral columns, and different asymmetries to opposing directions of a selected motion test (for example, right or left for the axial rotation test). Primary dysfunction asymmetries located in adjacent columns at the same segmental level were examined for similarity or dissimilarity in the direction of their limited mobilities. Three categories, as described previously, were used to classify the findings at each segmental level.

The observed frequency of Category III, linkage dysfunctions (existing as a similarity in the direction of restricted motion in two columns), and Category II, nonlinkage dysfunctions (dissimilarity in the direction of restricted motion in two columns) are reported for T9-12 in Table 3 . There is a statistically significant increase in the frequency of Category III dysfunctions present in renal patients. As judged by the chi square test for independent proportions, using one degree of freedom, no correction factor, and a one-tailed test, the probability of this increase occurring by chance is less than 1 percent. 


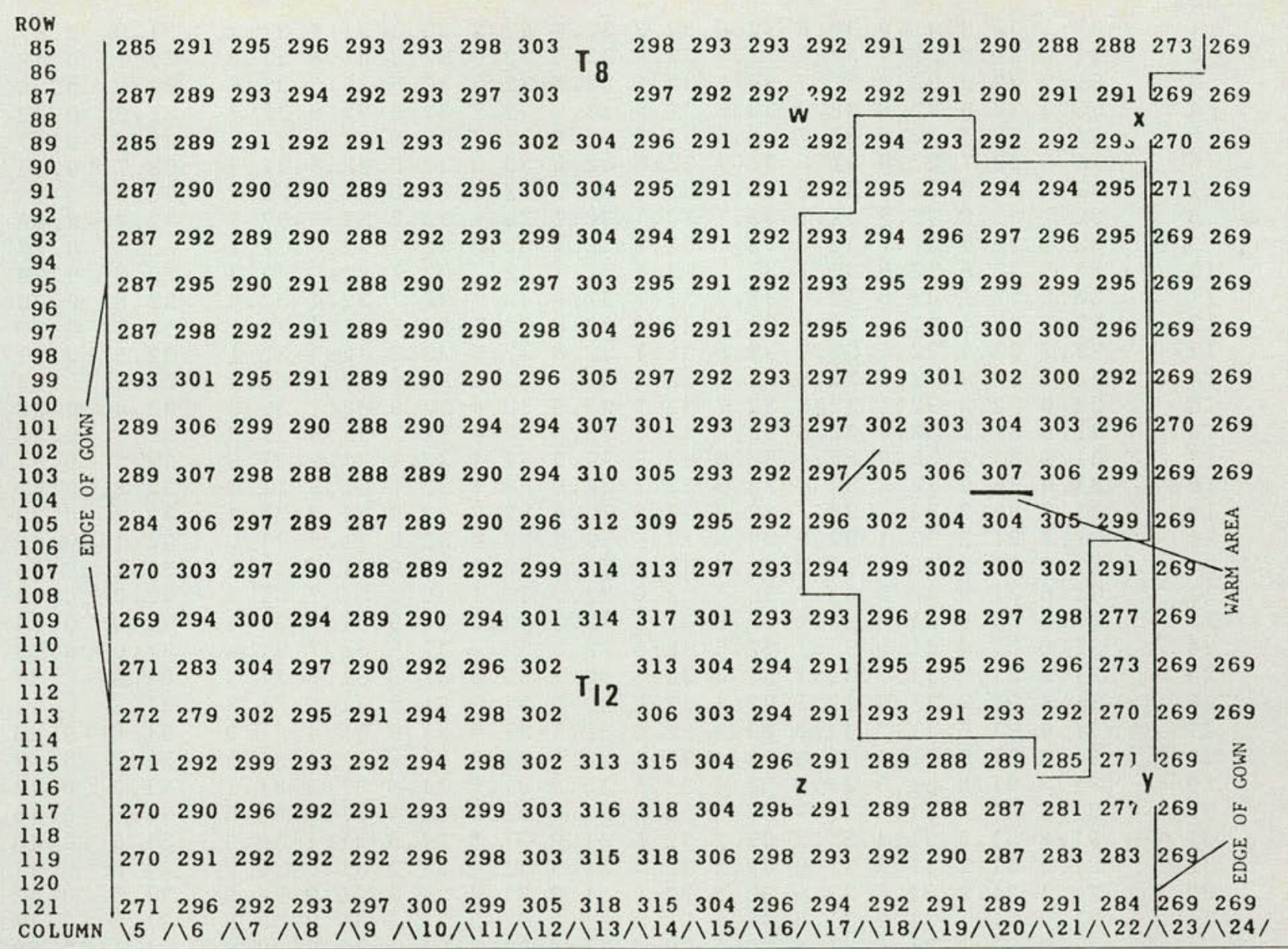

Fig. 3. Identification of a warm area in a partial thermogram of a renal dialysis subject. Pixels represent temperatures in degrees $C$. $x 10$ for the dorsal region of the back between T8 and T12. The $w, x, y, z$ symbols identify an area containing a warm area. The procedure used and the criteria for mapping the warm area included: (1) identifying a temperature difference greater than $0.5 \mathrm{C}$. between two adjacent pixels in a row (for example, row 103, column 17 and 18 [29.7/30.5]); (2) verifying that adjacent pixels have increased temperatures extending horizontally and vertically from the identified pixels; (3) drawing a border to include all pixels that exceed external pixel temperatures; and (4) verifying that pixel temperatures outside the border represent temperatures and temperature differences expected on the basis of patterns represented in the standard thermogram (Fig. 4). Note: The warm midline over the vertebrae probably represents venous drainage from warmer tissues.

\section{Skin temperature}

Thermographic-measured Tsk was used to estimate cutaneous blood flow, and we focused on warm areas that had an excess variance $>0.5 \mathrm{C}$. We assumed that renal disease would be associated with a renal-cutaneous-vasomotor reflex identifiable with increased skin temperature in the dorsal region T9-12. A visual procedure to identify warm areas in thermograms was developed using the following criteria: (1) a temperature difference $>0.5 \mathrm{C}$ between two adjacent pixels in a row identified a possible warm area; (2) the surrounding pixels indicated an increased temperature; (3) as the visual search extended horizontally and vertically, pixels would be found that represented expected skin temperatures and small differences in temperature be- tween pixels; and (4) a border, which included the warm pixels, could be identified as separating the interior warm pixels from adjacent exterior pixels with normal temperatures.

The procedure for visual analysis of thermograms is illustrated in Figure 3. A temperature difference of $0.8 \mathrm{C}$ between pixels in row 103 , columns 17 and 18 identifies a possible warm area. Adjacent pixels are noted to have similar high temperatures, which decrease both laterally and vertically. The pixels external to the border drawn around the warm area have temperatures around $29.2 \mathrm{C}$, consistent with other exterior pixels, and there are small temperature differences (around $0.1 \mathrm{C}$ ) between these external pixels. The warmest pixel within the borders, at $30.7 \mathrm{C}$, locates the 


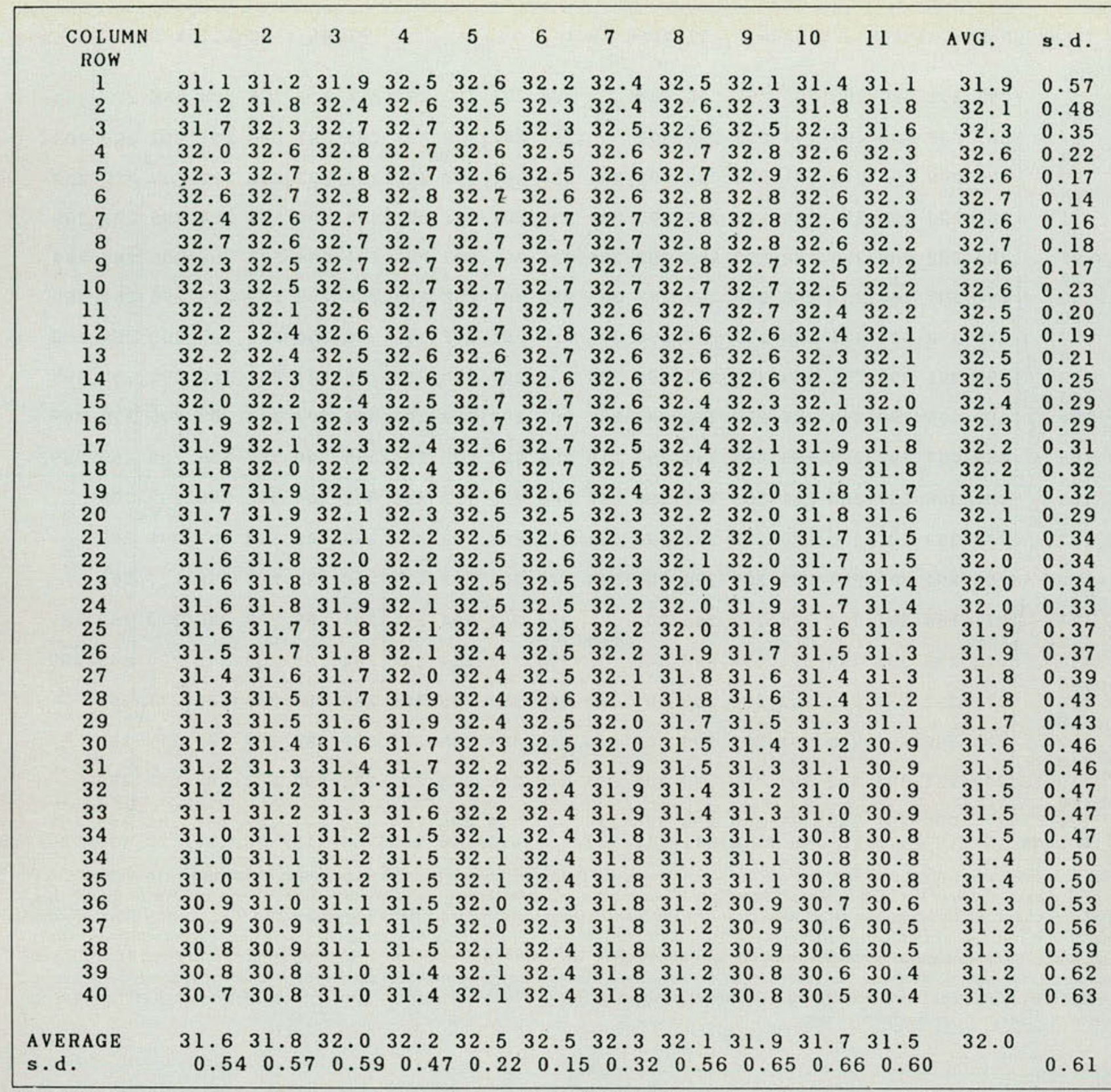

Fig. 4. Standard thermogram. Skin temperatures in the dorsal region T1 to T12 obtained from thermograms of 25 healthy subjects. Skin markers at T1 and T12 were aligned with upper and lower benchmarks on the thermograph videoscreen prior to taking thermograms. The 25 thermographic images were superimposed for mathematical analysis, which included calculation of the mean and variance for each set of 25 coinciding pixels. The means of 25 pixel temperatures were then used to construct the illustrated standard thermogram. The statistical data in the standard thermogram were calculated for the pixels in the figure. The variance between rows and columns of the standard thermogram identify the expected variance for that location and provide the criteria for identification of pixels with warmer than expected temperatures.

warm area at about the T11 vertebral level.

These criteria are supported by information from a standard thermogram ${ }^{16}$ (Fig. 4) and graphic analysis (Figs. 5A and 5B). The standard thermogram represents averaged data from thermograms of 25 normal subjects. Each pixel in the standard thermogram represents the average of pixels at the same location in the 25 thermograms. Averaging in this way was possible because of the technique used to obtain similarly sized images from all subjects. The means and variances for the entire thermogram and for each row and each column have been calculated from the standard thermogram, not from original data. The mean and variance for the 25 pixels at one location, eg. row 1 , column 1 , are not shown in Figure 4 but are used in statistical 

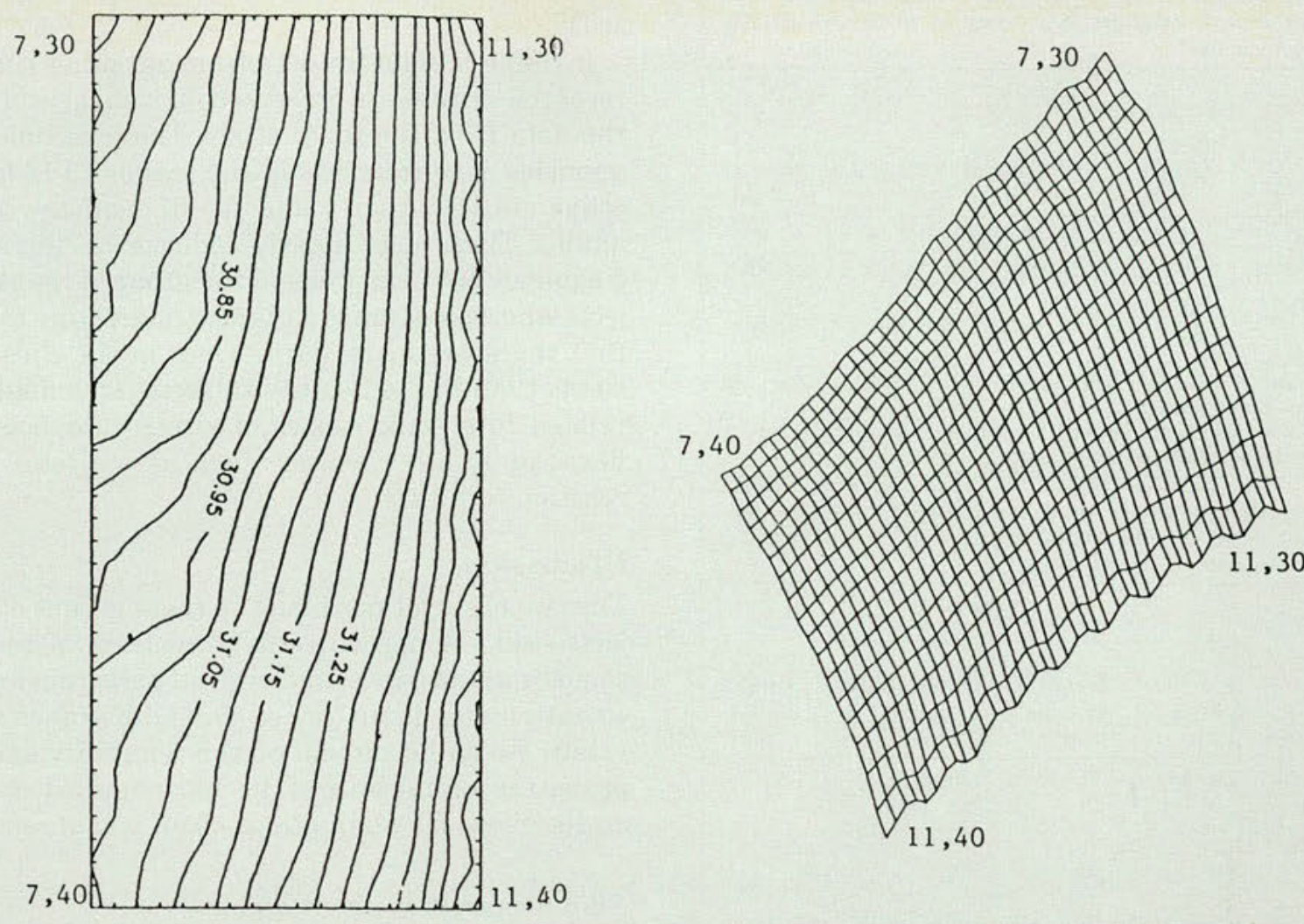

Figs. $5 A$ and $5 B$. Graphic analysis of standard thermogram for the dorsal region T8 to $T 12$ on the right. Data for the isothermal line (A) and temperature contour (B) graphs correspond to pixels in rows 30 to 40 and columns 7 to 11 in the standard thermogram (Fig. 4). Data for graphing have been calculated using least squares approximation and smoothing factor of 0.98 (Golden Software). Contours in graph $B$ are plotted from a point of view 45 degrees above the plane of the back, with the inferior border tilted 45 degrees from horizontal. The $x, y$ coordinates are indicated for the corners of the graphs. The graphs illustrate the uniformity of skin temperatures and small differences in temperatures between pixels.

comparison of thermograms with the standard thermogram.

The graphic plots in Figures $5 \mathrm{~A}$ and $5 \mathrm{~B}$ provide a means of visualizing the small temperature differences between isothermal lines and the uniform topographic surface representing Tsk. These plots, which represent pixels in rows 30 thru 40 and columns 7 thru 11 in the standard thermogram, Figure 4 , are for an area similar in size to those representing the warm area reported in results. Neither of the plots indicate large variations in Tsk between pixels.

The mean skin temperature, $32 \mathrm{C}$, in the standard thermogram, Figure 4, is higher than would be predicted for the resting nude or seminude subject with oral temperatures of $37 \pm 0.5 \mathrm{C}$ under the climate control conditions of an environmental temperature of $24 \mathrm{C}$ and 50 percent relative humidity. Clark and Edholm's ${ }^{17}$ discussion of the variables influencing skin temperature would suggest a skin temperature a few degrees above environ- mental temperature, i.e. about 26 to $28 \mathrm{C}$. The use of gowns open at the back and trousers to clothe our subjects partially explains the difference, but does not account for thermoregulatory responses in the cutaneous circulation of the back.

The standard thermogram identifies a temperature pattern, with the warmest area in the upper thoracic region, and temperatures decreasing toward the cervical region and inferiorly and laterally in the lower thorax. In Fig. 4, the outermost figures for standard deviation, ranging from 0.14 to $0.66 \mathrm{C}$., indicate that the variance in rows, columns, or complete thermogram are less than 0.1 C. This small temperature difference between pixels establishes the basis for visual examination of thermograms for temperature increases. Our criteria for statistically significant temperature difference between pixels, $>0.5 \mathrm{C}$., exceeds 3 times the standard deviation of any row or column in the standard thermogram.

The frequency of warm areas in the region T9-12 


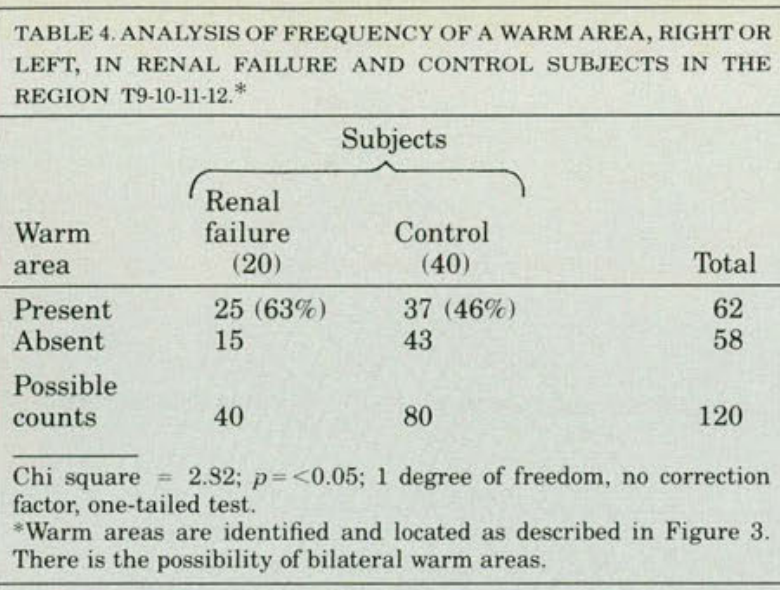

TABLE 5. COMPARISON OF RESULTS OF THERMOGRAPHIC MEASUREMENTS OF SKIN TEMPERATURE IN THE REGION T9-10-11-12 FOR RENAL AND OTHER STUDIES.*

\begin{tabular}{|c|c|c|c|c|}
\hline \multirow[b]{2}{*}{$\begin{array}{l}\text { Warm } \\
\text { area }\end{array}$} & \multicolumn{4}{|c|}{ Subjects } \\
\hline & $\begin{array}{r}\text { Renal } \\
\text { failure } \\
(20)\end{array}$ & $\begin{array}{l}\text { Renal } \\
\text { controls } \\
(40)\end{array}$ & $\begin{array}{l}\text { Other clinic } \\
\text { patients } \\
\text { (44) }\end{array}$ & $\begin{array}{r}\text { Healthy } \\
\text { young } \\
\text { adults } \\
(61)\end{array}$ \\
\hline Present & $25(63 \%)$ & $37(46 \%)$ & $26(30 \%)$ & $15(12 \%)$ \\
\hline Absent & 15 & 43 & 62 & 107 \\
\hline $\begin{array}{l}\text { Possible } \\
\text { counts }\end{array}$ & 40 & 80 & 88 & 122 \\
\hline
\end{tabular}

${ }^{*}$ Clinic subjects are subjects from other studies or ones not used in reporting on this study. Healthy subjects are osteopathic medical students without history of major health problems (surgery, illness, trauma, or current complaint).

in renal dialysis and control subjects was analyzed for statistical significance using the chi square test for proportions. This analysis is reported in Table 4 . The probability that the difference in frequency distribution of warm areas in the renal failure and control subjects could occur by chance was less than 5 percent.

A graphic method of analysis was used to verify the assumptions used in developing the visual method of identifying warm areas. The warm area identified in Figure 3 for the renal dialysis subject is presented in Figures $6 \mathrm{~A}$ and $6 \mathrm{~B}$ in plots of isothermal lines and temperature contours, with temperature as the $\mathrm{z}$-axis and location as the $\mathrm{x}, \mathrm{y}$-axis. These graphic plots support the manual method for identification of warm areas and also give the impression that the warm area has a horizontal axis corresponding to a dermatomal distribution of the increased temperatures. For comparison, the same contour and topographic plots are presented in Figure 5 for a corresponding region in the standard thermogram. The plots visually support the criteria of uniform temperatures and small temperature differences as the expected data to be used in analy- sis of a thermogram for locating and defining warm areas.

In Table 5, relevant data from our other curren research studies are presented for comparison with the data from the renal study. These include fre quencies of warm areas in the region T9-12 for 44 other clinic patients and for 61 healthy young adults. These data clearly indicate an increasec frequency of warm areas in the group of renal sub jects who were studied. It is also interesting to note that there are more warm areas in the clinic patients than in the healthy subjects. This might be related to specific causes of viscerocutaneous reflexes or to other causes, such as the total body reaction to illness.

\section{Discussion}

The two observations made on these groups of subjects-skin temperature as a measure of the vasomotor disturbance, and clinical palpation for the somatic motor disturbance-will be discussed separately. We will comment on the reliability and appraise the significance of the data obtained, as well as discuss some assumptions about spinal reflexes.

\section{Skin temperature observations}

According to technical specifications, the General Electric Spectrotherm 2000 has an accuracy of \pm $0.2 \mathrm{C}$. This degree of accuracy is achieved by liquid nitrogen cooling of the mercury-cadmium-teluride detector crystal, and by internal calibration for each scan line via reference to a calibration thermocouple. Accuracy is further enhanced by computer averaging of eight single measurements to reduce instrument noise effects and to provide smoothed data recorded as pixel temperature. Within a single thermogram, the differences in pixel temperatures are reliable to within $0.1 \mathrm{C}$. The differences between temperatures recorded in any two thermograms are reliable to within $0.2 \mathrm{C}$. when an external black-body radiant temperature standard is used as a reference temperature.

Measurements of Tsk are influenced by environmental heat exchange-radiant, evaporative, convective, and conductive - as well as conditions in the human subject. Cutaneous blood flow, local avascularity, local tissue metabolism, and sweating alter Tsk. Conducting the research in a climate control chamber reduces environmental influences and minimizes sweating. Equilibration for 20 or more minutes in the climate control chamber also reduces stresses that affect sympathetic motor control. Consequently, the use of the climate control chamber for thermographic measurement ensures that reflex control of cutaneous vasomotion becomes the independent variable, with other stimuli 


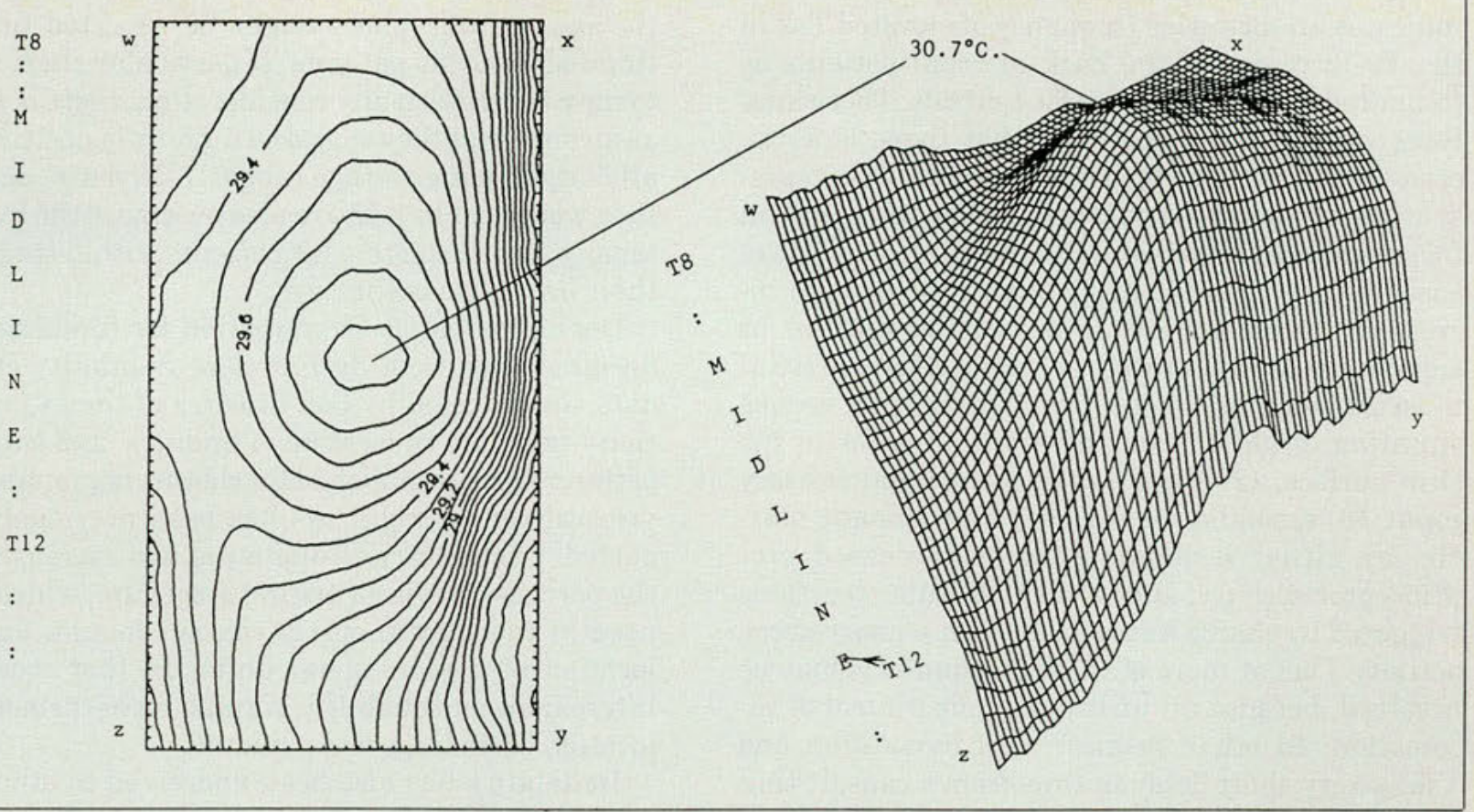

Fig. 6. Isothermal graphic analysis of a thermogram for a renal dialysis subject. Data for the isothermal line (A) and temperature contour (B) graphs correspond to data from pixels in the area bounded by $w, x, y, z$ in Figure 3. Data for the graphs have been calculated using least squares approximation and a smoothing factor of 0.98 (Golden Software*). Graph width-to-height ratio is 0.5. Contours in graph B are plotted from a point of view 45 degrees above the plane of the back, with the inferior border tilted 45 degrees from horizontal. The warmest skin temperature, $30.7 \mathrm{C}$, is indicated in both graphs. The warm area, located about vertebral level T11, is identified by the location of the warmest pixel. The area extends horizontally, suggesting a dermatomal orientation of the increased temperatures. Using criteria from the standard thermogram (Fig. 4), the borders of the graph suggest that the temperatures and temperature differences are similar to expected temperatures.

\section{minimized.}

There are a number of renal stimuli resulting from renal pathology that might account for the increased frequency of localized warm areas of Tsk in the lower dorsal region of T9-12. These stimuli or stimulus-like situations constitute the afferent limb of the renal to cutaneous blood vessel reflex, which accounts for vasodilation and, therefore, warm skin. This vasodilation is either active, as a result of sympathetic activity, or passive, as a result of inhibited vasoconstrictor tone.

The stimuli in the renal dialysis patient are related to sequelae of renal failure and may be the result of any of three complications, that is, ischemia, intra- and extra-cellular environmental changes within the kidney, and, in more severe complications, obliteration of renal afferent nerve impulses. Renal ischemia is a strong nociceptor stimulus. Also, the changes in composition of extracellular fluid that accompany deterioration of renal tissues create a number of chemical stimuli that are capable of initiating impulses in the C-type nerve fibers. Severe tissue damage or nephrectomy ablates afferent nerve activity, with the implication, according to the "gate" theory, that the absence of afferent renal impulses allows the nonrenal sensory input to contribute to facilitation.

We assume that these stimuli arising from the kidney (including in this case, decreased stimuli) will result in a reflexly initiated change in localized vasoconstrictor activity in skin, which in turn will create the altered Tsk. This assumption of discrete sympathetic mediated response is supported by observations in other studies. ${ }^{18}$ The classic concept of an integrated or generalized sympathetic nervous response is in conflict with our proposed discrete control of sympathetic nerve fibers. The creation of warm areas (passive vasodilation) is consistent, however, with other observations ${ }^{19}$ of "pain"-induced vasodilation in patients with low-back pain or arthritic knees. It appears reasonable to assume that the increased frequency of warm areas in the lower back, T9-12 region represents a renocutaneous reflex, with an afferent limb from a diseased kidney and an efferent limb that effects the inhibition of cutaneous vasoconstrictor activity. 
The thermographic pattern analysis (Table 4) indicates an increased frequency of elevated Tsk in the T9-12 region of the back in renal patients as compared with frequencies for controls. These analyses support our hypothesis that there is a viscerosomatic reflex, with the elevated Tsk representing an increased cutaneous blood flow and an increased heat transfer occurring in the regions of vasodilation. Possible mechanisms include: (1) increased muscle activity in paraspinal muscles or segmentally innervated portions of superficial muscles, such as trigger-point areas with venous shunting of blood from the active muscles to the skin surface; (2) vasodilation from renal sensory input; (3) vasodilation initiated from somatic muscle, by either ischemic pain or increased proprioceptor activity; and (4) local vascular reactions triggered by changes associated with sensory nerve activity. One or more of these mechanisms may be involved, because all utilize nervous control of vasomotion. In other studies ${ }^{20,21}$ of circulation and Tsk, a very short delay in time from a causal stimulus to the vasomotor reaction was observed, suggesting a nervous-mediated response.

\section{Clinical observations}

The clinical diagnosis, by which assignment of patients to groups was made, reflects accepted diagnostic standards. Criteria for exclusion from the study were based upon data in patient medical records. For inclusion in the renal group, patients were accepted from the dialysis unit. All renal patients had a history of hypertension followed by failure of renal function, as evidenced by increased serum metabolite levels and progression to dependence on dialysis as essential for life maintenance. Patients in the renal group had many different causal and contributing factors; however, the presence of renal failure was well documented. For the hypertensive and normotensive groups, our criteria clearly eliminated borderline, unstable, or mild hypertensives from either group. Some normotensives were excluded from the study on the basis of new information at the time of their examination; other normotensives, as mentioned previously, had been inadequately screened, proved to be hypertensive on interview, and, when eligible, were retained in the larger pool of patients meeting the criteria for hypertension.

The procedures to minimize examiner bias received considerable attention. All response variables were measured and recorded before the patient left the climate control chamber. Our examination procedures were carefully standardized for recording only thoracic segmental dysfunction, and the opportunity was limited to observe clues that related to the general condition of the patient. Only the most subtle clues might be expected to distinguish dialysis patients. If particular clues were to have been given any consideration, there is little assurance that they would be uniformly positive for all dialysis patients (age range 27-75 years), or that they would not be positive also in some of the hypertensive and normotensive patients within stages of their own serious illnesses.

During palpatory examination for fundamental 3 -segment units of dysfunction, reliability of the data was affected by two features of the examination-accuracy in location of findings, and validity of the criteria established for classifying findings of visceral reflex origin. As has been previously reported,${ }^{10}$ reliability of diagnosis was increased by the percussion type of testing procedure, which was used in the central spinal column to scan for the location of dysfunctional units. In that study of interexaminer reliability, significant agreement on location of findings was achieved.

Reliability has also been addressed in other interexaminer studies, ${ }^{13,22}$ which were conducted to test agreement of trained examiners in their use of segmental motion tests to make diagnostic decisions of segmental dysfunction. Data about the directions of a segment's resistance to specified motion tests provide precise descriptors of the motion asymmetries present in a given segment. Specific tests and recordings for obtaining these data require trained examiners. In principle, this is similar to training for psychomotor skill performance and applies to many procedures carried out in a research setting. The interexaminer studies that tested reliability in the use of palpatory testing procedures utilized third-year medical students without extensive clinical experience. Their observations related to positive findings were reproducible because of training to conduct tests and to make judgments based upon defined criteria for positive findings.

Our classification of segmental dysfunctions into categories I, II, and III was derived from the clinical use of the specific directions of an individual segment's motion asymmetries to recognize differences in the configuration of dysfunctional units in the thoracic vertebral and costal columns. Procedurally, in the investigation of a dysfunctional segment, the examiner uses fingers of one hand to monitor by palpation the immediate response to a gross passive motion test, which is being introduced simultaneously by the other hand. Judgment on the character of a specific asymmetry is made by comparing responses to opposing directions of motion at the same segment; the directions of immediately increasing resistance are used to 
characterize the asymmetry. For the current study, we used motion tests for resistance to passive shoulder rotation (right or left), shoulder sidebending (right or left), and resistance to active respiration (inhalation or exhalation) to evaluate an individual segmental motion asymmetry. Also, the motion tests were repeated at the segments above and below the primary segment to confirm/deny the presence of mirror-image resistances to motion, as a method for establishing the reliability of a dysfunctional unit. Its location was recorded only when the findings of a primary segment's resistance to motion were confirmed by the presence of opposing directions of motion resistance in the two adjacent (superior and inferior) segments. The same three motion tests also were used to examine for resistance to motion in the vertical columns of costal segments to the right and left of the midline vertebral column. Each column of costal segments was examined for the presence of dysfunctional units, again by using resistance to motion in adjacent costal segments (superior and inferior) to assure reliability of location of the primary dysfunctional costal segment. When primary dysfunctional segments were identified in the midline and in a lateral costal column at the same spinal level, comparisons were made of their similarities of resistance to motion (direction) during all three motion tests. In this manner, a basis was established for making judgments regarding presence of the linkage effect that was evident when two adjacent dysfunctional units (one vertebral and one costal) showed identical asymmetries, thus distinguishing a category III finding from category II when adjacent column dysfunctions are dissimilar.

Finally, the technique of recording palpable findings on a cloth record that overlies the actual primary segmental level of the finding assured that its relation to designated spinous landmarks was accurate rather than judgmental. The same landmarks were used for deriving the location of excess temperature variances during analysis.

The clinical observations of some osteopathic authors $^{23-27}$ of palpable musculoskeletal findings in patients with visceral disease have tended to affirm longstanding assumptions that viscerosomatic reflexes contribute significantly to clinical diagnosis. This clinical impression was supported by Kelso's ${ }^{28}$ study on the incidence and location of palpable findings in patients hospitalized with a variety of diagnosed diseases. Also supported by clinical experience is the positive feedback nature of the visceral reflex disturbance on somatic motor function; findings in the somatic system associated with visceral disease are often self-maintained, even after the visceral source is alleviated or surgically re- moved. The goal in a manipulative approach has been to interrupt the disturbed cycle of spinal reflexes in facilitated nerve pathways created by specific visceral sensory inputs at dysfunctional spinal segments. By addressing the neuromuscular component, the factors of local increased muscular tension, positional distortion, and asymmetric response to motion are reduced as a source of continuing bombardment of afferent impulses at these segments during posture and movement. There may be similar beneficial influences from changes in nervous control of circulation after manipulative treatment of segmental somatic dysfunction.

There is an anatomic basis for the hypothesis that somatic manifestations of renal disease are expected to occur in the thoracic spinal region T9-12. Although hypertension is an early concomitant condition with primary renal disease, the presence of renal disease that is secondary to benign essential hypertension is delayed; it usually presents after a prolonged period of chronically elevated blood pressure. If hypertension is of recent origin, if laboratory tests of impaired renal function are within normal limits, and if there is absence of visceral sensory reflex manifestations at T9-12, there is basis for considering other than renal factors in the classification of hypertensive disease. The presence of somatic manifestations (T9-12) provides supporting evidence that renal complications may be developing. In this spinal region, however, differential diagnosis must consider somatic manifestations of somatic reflex origin, as well as those of visceral origin from other than renal causes. In our study, it was necessary to review data in the patient's medical record to control for these variables in the selection process.

\section{Conclusion}

Either somatic motor or skin temperature changes might be observed singularly in this type of research. Both were included in this controlled study so as to examine two aspects of viscerosomatic reflex activity simultaneously in the same sampie. The increased frequency of both somatic motor and skin temperature changes in the T9-12 region for the renal failure group supports the presence of viscerosomatic influence in renal disease and indicates two reflex responses that may be present in somatic tissues.

\section{Addendum}

A mobile segment is represented by a bony unit with articular surfaces for movement, and the adnexal tissues that (1) move it, (2) allow it to be moved from one position to another, and (3) stabilize it in one position. Criteria for norm of a 
mobile unit at rest include palpable qualities of symmetry of structural position and symmetry of soft-tissue tone, both superficial and deep. During tests for gross physiologic movement, using passive rotations and translations and active respiration, the criteria are a palpable sense of symmetry and a midpoint (of range of motion); an initial easy compliance in opposing directions expresses smooth adaptive behavior of segmental tissues (both bony and soft) before encountering the eventual sense of increasing resistance (equidistant) at the end points of a segment's range of motion.

Palpatory criteria for segmental dysfunction consist of the following characteristics: (1) a palpable sense of bony positional irregularity/asymmetry; (2) a palpable sense of increased (and asymmetric) resistance to pressure of superficial and deep tissues, locally at a segment, when compared with surrounding segments; and (3) a palpable sense of asymmetry in local segmental response to motion tests in opposing directions. First, in response to some directions of motion tests, the expected sense of initial compliance by the segment is lost, and it is replaced by a sense of immediately increasing resistance (palpable increase in soft-tissue tension and decreased mobility) in the direction of the test. This sense of resistance continues to increase sharply toward a firm end point of range, which is encountered sooner than in the opposing direction. In the opposite direction, the immediate response is an increasingly easy compliance, which gradually dissipates as range is continued toward a normal sense of end point. This asymmetric response pattern of immediately increasing resistance and immediately increasing compliance in opposing directions of a motion test is descriptive of the response-to-motion characteristics at a dysfunctional mobile segment. It is a consistent (predictable) feature of local response to all aspects of physiologic motion tests (rotatory, translatory, and respiratory) at that segment.

This study was initiated during sabbatical leave (WLJ). The assistance of the Chicago College of Osteopathic Medicine in providing facilities and patients for this study is greatly appreciated. The assistance of Drs. Mayer L. Horensten, Robert E. Kappler, Mark H. LaBeau, and Neil Natkow is acknowledged.

1. Warwick, R., and Williams, P.L.: Gray's Anatomy. Brit. Ed. 35. W.B. Saunders Co., Philadelphia, 1973

2. Pottenger, F.M.: Symptoms of visceral disease. Ed. 5. C.V. Mosby Co., St. Louis, 1938

3. Guyton, A.C., et al.: A systems analysis approach to understanding the long-range arterial blood pressure control and hypertension. Circ Res 35:159-76, Aug 74

4. Netter, F.H.: Part 1. Anatomy and physiology. In The CIBA collection of medical illustrations: Volume 1, Nervous system. CIBA Pharmaceutical
Company, West Caldwell, New Jersey, 1983

5. Eble, J.N.: Somatic manifestation of experimental visceral disturbances. J Osteopathy 65:13-6, Jan 58

6. Coldwater, K.B., et al.: Sweating patterns and vascular responses in the lower extremity in man elicited by stimulation of the sympathetic trunk. Surgical Forum 53:486-92, Jan-Jun 53

7. Ruch, T.C., and Patton, H.D.: Physiology and biophysics. Ed. 19. W.B. Saunders Co., Philadelphia, 1965

8. Kuntz, A.: Autonomic nervous system. Ed. 3. Lea and Febiger, Philadelphia, 1945

9. Bannister, R.: Testing autonomic reflexes. In Autonomic failure, edited by R. Bannister. Oxford University Press, New York, 1983

10. Johnston, W.L., et al.: Interexaminer study of palpation in detecting location of spinal segmental dysfunction. JAOA 82:839-45, Jul 83

11. Kelso, A.F., Grant, R.G., and Johnston, W.L.: Use of thermograms to support assessment of somatic dysfunction or effects of osteopathic manipulative treatment. Preliminary report. JAOA 82:182-8, Nov 82

12. Johnston, W.L., and Hill, J.L.: Spinal segmental dysfunction. Incidence in cervicothoracic region. JAOA 81:67-76, Sep 81

13. Johnston, W.L., et al.: Palpatory findings in the cervicothoracic region. Variations in normotensive and hypertensive subjects. A preliminary report. JAOA 79:300-8, Jan 80

14. Johnston, W.L.: Interexaminer reliability studies. Spanning a gap in medical research-Louisa Burns Memorial Lecture. JAOA 81:819-29, Aug 82

15. Dinnar, U., et al.: Classification of diagnostic tests used with osteopathic manipulation. JAOA 79:451-5, Mar 80

16. Johnston, W.L., and Kelso, A.F.: Use of thermograms to assist in identification of significant warm or cool areas in records of skin temperature on the back in human subjects (abstract). JAOA 85:601-2, Sep 85 17. Clark, R.P., and Edholm, O.G.: Man and his thermal environment. Edward Arnold Publishing, Ltd., London 1985, pp.46-68, 155-171

18. Barman, S.M.: Spinal cord control of the cardiovascular system. In Nervous control of cardiovascular function, edited by W.C. Randall. Oxford University Press, New York, 1984

19. Woodrough, R.E.: Medical infrared thermography. Principles and practice. Cambridge University Press, New York, 1982

20. Larson, N.J., Walton, M.W., and Kelso, A.F.: Effectiveness of manipulative treatment for paresthesias with peripheral nerve involvement (abstract). JAOA 80:216, Nov 80

21. Hallihan, M.R., et al.: A prospective clinical study on the efficacy of osteopathic manipulative treatment for Bell's palsy patients (abstract). JAOA $84: 75$, Sep 84

22. Johnston, W.L., et al:: Identification of stable somatic findings in hypertensive subjects by trained examiners using palpatory examination. JAOA $81: 830-6$, Aug 82

23. Larson, N.J.: Manipulative care before and after surgery. Osteopathic Med, pp. 41-9, Jan 77

24. Larson, N.J.: Summary of site and occurrence of paraspinal soft tissue changes of patients in the intensive care unit. JAOA 75:840-2, May 76 25. Johnston, W.L.: Segmental behavior during motion. III. Extending behavioral boundaries. JAOA 72:462-75, Jan 73

26. Beal, M.C.: Palpatory testing for somatic dysfunction in patients with cardiovascular disease. JAOA 82:822-31, Jul 83

27. Beal, M.C., and Kleiber, G.E.: Somatic dysfunction as a predictor of coronary artery disease. JAOA 85:302-7, May 85

28. Kelso, A.F.: A double blind clinical study of osteopathic findings in hospital patients. Progress report. JAOA 70:570-92, Feb 71

Accepted for publication in July 1986.

Dr. Johnston is a Professor in the Department of Family Medicine at Michigan State University, College of Osteopathic Medicine, East Lansing. Dr. Kelso is a Research Professor in the Department of Osteopathic Medicine, Dr. Hollandsworth is an Associate Professor in the Department of Internal Medicine and Director of the Section of Nephrology, and Dr. Karrat is an Associate Professor in the Department of Family Medicine and Director of the Residency Training Program of Family Medicine at Chicago College of Osteopathic Medicine.

Dr. Johnston, Department of Family Medicine, MSU-COM, East Lansing, Michigan 48824. 